\title{
Zur Pathologie des Ligamentum rotundum uteri.
}

Von

Dr. Agnes Bluhm in Berlin.

(Mit 3 Abbildungen auf Tafel XXVII.)

"Unsere Kenntniss von der Pathologie des Ligamentum rotundum beruht zur Zeit noch auf so wenig zahlreichen Berichten, dass es gewiss erlaubt erscheint, auch einzelne Mittheilungen über einschlägige Fälle zur Veröffentlichung zu bringen".

Wenn auch bereits 6 Jahre vergangen sind, seitdem A. Martin ${ }^{1}$ ) seine gleichnamige Publication mit obigen Worten begründete, so haben dieselben, heute noch vollauf Geltung und mögen zur Rechtfertigung auch der folgenden Mittheilung dienen.

Am 3. December 1895 consultirte mich die 20 jährige unverheirathete Putzmacherin K. mit der Angabe, dass sie seit 8 Tagen Schmerz in der rechten Leistengegend verspüre, wo sich eine kleine Geschwulst gebildet habe, die ihr früher nicht aufgefallen sei. Eine äussere Ursache für ihr Leiden wusste sie nicht anzugeben.

Stat. praes.: Ich constatirte medianwärts vom Annulus inguinalis int. dext., dem horizontalen Schambeinast aufliegend, einen pflaungrossen Tumor von derb-elastischer Consistenz. Aeussere Haut darüber verschieblich, nicht geröthet. Tumor etwas beweglich, scheint durch einen Strang mit dem Leistencanal in Verbindung zu stehen; wenigstens fühlt man, dass bei Verschiebung der Geschwulst zur Symphyse hin sich ein Strang, der sich von ersterer bis zum Leistenring verfolgen lässt, anspannt. Reposition des Tumors durch den Inguinalcanal in die Bauchhöhle gelingt nicht. Bei Hustenstössen vermehrt sich sein Volumen ebenso wenig, wie es sich durch Druck vermindern lässt. Percussionsschall über der Geschwulst gedämpft. Betastung mässig empfindlich. Die Genitaluntersuchung ergiebt einen anteflectirten, dabei in toto reclinirten Uterus; linke Adnexe ziemlich deutlich, rechte nicht deutlich fühlbar. Auch vom Rectum aus lässt sich das rechte Ovarium nicht mit Sicherheit nachweisen.

Diagnose: Eine Lymphdrüsenschwellung, sowie eine einfache Hernia inguinalis waren nach obigem Befunde wohl auszuschliessen.

1) Zeitschr. f. Geb. u. Gyn. Bd. XXII. 1891. 
Somit blieb nur die Annahme einer Hernia inguinalis mit dem cystisch-degenerirten Ovarium als Inhalt oder eines Tumor des Lig. rot. übrig. Wegen des nicht sicher zu palpirenden rechten Ovariums musste die Diagnose zwischen den letzten beiden Möglichkeiten offen bleiben.

Therapie: Zunächst hydropathische Umschläge, welche die Schmerzen bald linderten und später, da Pat. "etwas zum Finreiben" wünschte, Ung. Kal. jodat., wonach sich die Geschwulst verkleinert haben soll.

Weiterer Verlauf: Im November 1896 erschien Pat. wieder bei mir mit der Angabe, dass der Schmerz, der inzwischen nur während der Menstruation aufgetreten sei, sich jetzt häufig auch ausserhalb derselben einstelle und sie am Arbeiten hindere. Auch schwelle die Geschwulst stets kurz vor der Periode an, um im Verlauf derselben wieder kleiner zu werden. Von der Richtigkeit dieser Angabe konnte ich mich alsbald persönlich überzeugen. Es schien dieses Symptom zu Gunsten einer Ovarialhernie zu sprechen. Mit Rücksicht auf die häufig wiederkehrenden Schmerzen und die dadurch bedingte Arbeitsbehinderung schlug ich Pat. die Exstirpation des Tumors vor. Sie konnte sich dazu aber erst entschliessen, als nach einem Fall Anfang Februar 1897 der Schmerz constant wurde und sie am Gehen hinderte. Auch war der Tumor inzwischen gewachsen.

Operation am 16. März 1897. Vor Beginn derselben Genitaluntersuchung in Narcose, die, da sie mit Rücksicht auf den intacten Hymen mit einem Finger ausgeführt werden muss, keine neuen Anhaltspunkte für die Diagnose ergiebt. Rechte Adnexe ganz undeutlich fühlbar; Bewegungen des Uterus, die beschränkt sind, üben auf die Lage des Tumors keinen Einfluss. Tumor selbst nach der vorausgegangenen 24 stündigen Bettruhe etwas abgeschwollen. Hautschnitt $6 \frac{1}{2} \mathrm{~cm}$ lang in der Längsachse des Tumors, $d$. h. in schiefem Winkel zum horizontalen Schambeinast. Nach Durchtrennung des subcutanen Fettgewebes stösst man auf den Tumor, der in ein lockeres gefäss- und fettreiches Bindegewebe eingehüllt ist. Freilegung des Annul. ing. int.; in demselben erscheint das ca. $4 \mathrm{~mm}$ dicke Lig. rot., das in die Geschwulst übergeht. Medianwärts gehen aus derselben strahlenförmig derbe Bindegewebsbündel hervor, die sich in der Gegend des Mons veneris ansetzen. Die bindegewebige Hülle des Tumors lässt sich an einer Stelle lospräpariren, und es tritt hier sich vorwölbend ein etwas über kirschkerngrosses Cystchen zu Tage, das bei Berührung platzt und einen blutig-serösen Inhalt entleert. Eine weitere Ausschälung des Tumors aus seiner Hülle gelingt nicht. Es folgt nun die Isolirung desselben von seiner Unterlage (Bauchmuskelfascie) und die Durchtrennung des Lig. rot. an der medianen und lateralen Seite der Geschwulst. Dabei zeigt dasselbe dort, wo es sich als runder Strang präsentirt, einen centralen Canal, der sich einerseits in eine Cyste des Tumor's fortsetzt, andererseits sich $11 / 2 \mathrm{~cm}$ weit mit der Sonde in den Inguinalcanal hinein verfolgen lässt. $\mathrm{Da}$ sich das Lig. nicht soweit aus dem Leistenring herausziehen lässt, dass man seine beiden Enden, zwischen denen der Tumor eingeschaltet war, vereinigen konnte, wird sein zum Uterus verlaufender Theil mit 2 Catgutfäden am Annul. ing. fixirt. Schluss der Hautwunde durch Seidensuturen, die nach 4 Tagen entfernt werden.

Am 10. Tage wird Pat. gesund entlassen. Die Beschwerden haben seitdem vollkommen aufgẹhört. 
Makroskopischer Befund: Der Tumor, der nach seiner Exstirpation Pflaumengrösse besitzt, zeigt auf dem Durchschnitt zahlreiche theils kugelige, theils schlauchförmige, stecknadelkopf- bis kirschkerngrosse Hohlräume, die zum grösseren Theil mit einander communiciren und einen blutig tingirten serösen Inhalt haben. Die Septa sind mässig derb; an einer kleinen Stelle, die sich durch eine weissliche Farbe auszeichnet, ist die Substanz fast knorpelhart.

Mikroskopischer Befund: Mikroskopisch erweist sich die Geschwulst als ein Cystofibrom, das in eine schmale Kapsel von fett- und gefässreichem lockeren Zellgewebe eingeschlossen ist. Letztere enthält vereinzelte Bündel quergestreifter Muskelfasern. Das die Grundsubstanz des Tumors bildende Bindegewebe ist fibrillär und zellarm. Es zeigt keine regelmässige Anordnung; die einzelnen Züge verflechten sich vielfach mit einander; doch entspricht im Grossen und Ganzen der Faserverlauf der Längsachse des Tumors. Hie und da sieht man kleinere Züge glatter Muskelfasern. Die Vascularisation ist eine reiche und es fällt an den kleinen Arterien die starke Ausbildung der Muscularis auf. Die an dem frischen Präparat dem unbewaffneten Auge glattwandig erschienenen kleinen Cysten (Fig. $1 \mathrm{~L}$ ) zeigen auf dem mikroskopischen Schnitt vielfache Ausbuchtungen. In die Buchten münden schmale gewundene und verzweigte Gänge ein. Daneben findet sich eine grosse Anzahl ähnlicher, mehr gestreckter Hohlräume (Fig. $1 \mathrm{~L}^{\prime}$ ) mit reichlichen Ausbuchtungen und Verästelungen von sehr variablem Kaliber und oft auffallender Länge. Zuweilen treten mehrere derselben radiär zusammen, wie überbaupt das Ganze sich als ein Netzwerk vielfach gebuchteter, engerer oder weiterer anastomosirender Canäle darstellt. Im grossen Ganzen lässt sich aber auch hier die Tendenz der Streckung entsprechend der Längsachse des Tumors erkennen. Die Wand der grösseren Hohlräume ist zum Theil - wahrscheinlich durch mechanische Verletzung bei der Präparation - ihrer Bekleidung beraubt. Wo dieselbe erhalten ist, erweist sie sich als ein flaches Endothel; in den Buchten gewinnt dasselbe an Höhe, um am blinden Ende der einmündenden Gänge bisweilen niedrig-cylindrisch zu werden. Die kleineren Hohlräume sind meist mit kubischen Zellen, deren freier Rand häufig kuppelförmig abgerundet ist, ausgekleidet. Ueberall sitzen diese Zellen der fibrillären kernarmen und durchscheinenden Grundsubstanz der Geschwulst innig auf. Die Lumina aller dieser Abschnitte des verzweigten Systems sind leer. Hin und wieder lagern dem flachen Randbelag Pigmentmassen an. In unmittelbarer Nachbarschaft mancher Blutgefässe und der ektatischen Räume finden sich Anhäufungen von Rundzellen. Während dieselben die Gefässe in ziemlich gleichmässiger Schicht umgeben, bilden sie an den erweiterten Spalten vielfach knotenartige, in das Lumen derselben hineinragende Vorsprünge. Ausser zahlreichen Capillaren bergen diese Rundzellenanhäufungen ins Gewebe ausgetretene rothe Blutkörperchen und reichlich gelbliches bis tief rostbraunes Pigment, theils frei, theils in grosse Zellen eingeschlossen. Auch das die Räume an dieser Stelle auskleidende Endothel ist häufig pigmentirt.

Neben diesen zweifelsohne als Lymphangiektasien zu deutenden Gebilden findet sich eine $\mathrm{zweite}$ Gruppe wohlcharakterisirter Hohlräume, die auf den ersten Blick als Drüsen imponiren. Sie sind bei weitem spärlicher vertreten als die ersteren und zumeist im Querschnitt getroffen. Derselbe ist theils kreisrund (Fig. $1 \mathrm{D}$ ), theils halbmond- 


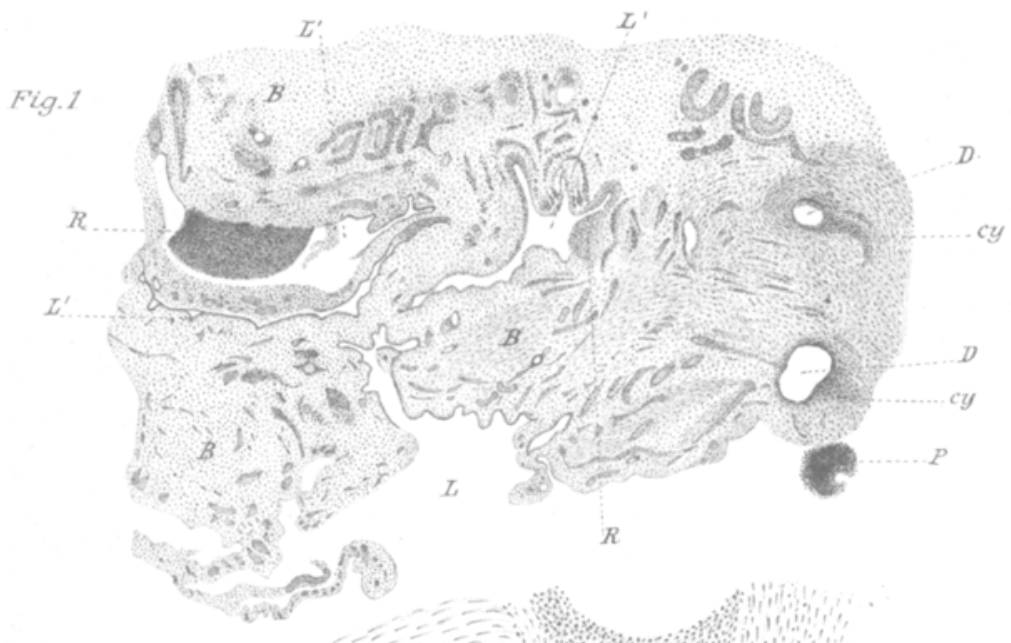

Fig. 2.
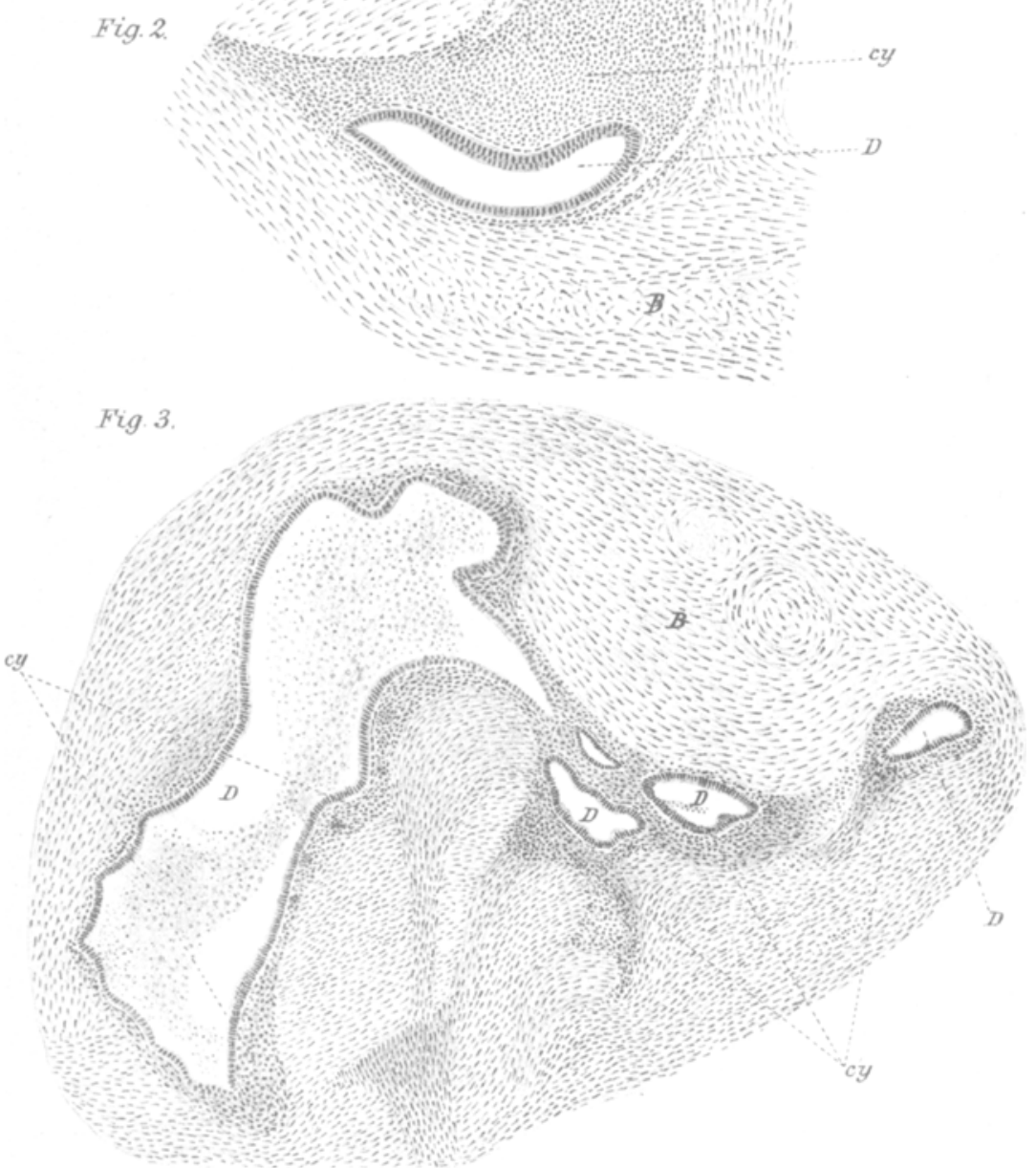
förmig (Fig. II D) und durch eine Anzahl von Schnitten zu verfolgen. Einmal trifft man eine grössere Gruppe von Drüsen beisammen, die eine unregelmässige Configuration im Vergleich zu einander zeigen (Fig. III).

Was alle diese drüsigen Gebilde von den obengeschilderten Lymphgängen unterscheidet, ist zunächst ihre zellige Auskleidung. Sie besitzen ein ziemlich hohes pallisadenförmiges Cylinderepithel mit grossem bläschenförmigen Kern und leicht gekörntem Plasma. Flimmerhaare lassen sich - das Präparat wurde nicht frisch untersucht - nicht mit Sicherheit nachweisen, sondern an mehreren Stellen nur vermuthen. Zweitens ruhen diese Epithelien nicht direct auf der nackten kernarmen bindegewebigen Grundsubstanz des Tumors, sondern sind von derselben scharf geschieden durch ein Stroma, das stellenweise durchaus der Tunica propria der Uterusschleimhaut gleicht (Fig. II). Es ist breiter oder schmaler, gegen die umgebende kernarme fibrilläre Substanz nicht scharf abgegrenzt und zeigt zuweilen insofern eine besondere Anordnung, als es sich an den halbmondförmig gebogenen Bildungen ausschliesslich oder vorwiegend in die Concavität hineinragend findet (Fig. II cy). Es wird dadurch der Eindruck hervorgerufen, als ob die Gestalt der Drüse durch das halbkugelige Vorspringen eines Rundzellenknotens in ihr Lumen bedingt sei. Dabei ist das Epithel an dieser Seite etwas höher als an der convexen. Auch an den Drüsen finden sich inmitten der Rundzellenanhäufungen $\mathrm{Ca}$ pillaren, freie Blutkörperchen und Pigment, wenn auch nicht so reichlich wie an den Lymphgefässen. Zuweilen haben sich die Drüsen von ihrer zellreichen Umscheidung retrahirt, etwa so wie man das an Uterusdrüsen zu sehen gewohnt ist.

In dem Lumen der Drüsen liegen Conglomerate von Detritus, schwach färbbaren weissen und rothen Blutkörperchen und einzelnen grossen, eigenthümlich glänzenden blassen Zellen mit feinkörnigem Plasma und einem grossen oder mehreren kleineren Kernen.

Wie aus dem mikroskopischen Bilde erhellt, haben wir es im vorliegenden. Fall mit einem lymphangiektatischen Cystofibrom des Ligamentum rotundum zu thun, ein Befund, der nicht allzu selten ist ${ }^{1}$. Was in unserem Fall wesentlich interessirt sind die drüsenartigen Gebilde, welche wir einzeln oder selbst gruppirt mit cystogener Einscheidung und wohlcharakterisirtem Epithel als Cystofibromeinschlüsse vorfanden. Es ist von vornherein einleuchtend, dass es sich hier um in der Fötalzeit verschleppte Keime aus einer Partie des Genitalapparates handelt. Woher stammen diese Kejme?

1) Anmerk.: Nebenbei sei bemerkt, dass der in meinem sowohl als in manchen anderen Fällen sonderbare Canal des Ligaments, der sich in das Cystom fortsetzte, mir nicht ein nichtobliterirter fötaler Canal (Weber'sche Hypothese), sondern ein einfacher Lymphspalt zu sein scheint. Da das Ligament sich nicht sehr weit hervorziehen liess, so wurde auf Exstirpation eines Stückes desselbēn verzichtet. 
Sehen wir uns in der Literatur nach analogen Fällen um, so finden wir zwei, die sich zum Vergleich heranziehen lassen. Der eine ist von Cullen im John Hopkins Bulletin, Mai und Juni 1896, beschrieben, der andere wurde von Pfannenstiel auf der VII. Versammling der deutschen Gesellschaft für Gynäkologie in Leipzig im Juni 1897 demonstrirt.

In dem Fall von Cullen, der klinisch viel Aehnlichkeit mit dem meinigen zeigt, handelt es sich um ein gefäss- und pigmentreiches Myom, das in allen Richtungen von Drüsen durchzogen ist. Diese Drüsen sind von ähnlichem Stroma umgeben wie das der Mucosa uteri. Einige enthalten runde Massen von Protoplasma, in welchen mehrere Kerne verstreut sind. "An manchen Stellen zeigen die Drüsen eine eigenthümliche Anordnung und stimmen mit v. Recklinghausen's ${ }^{1}$ ) "Pseudoglomeruli" " überein. Bekanntlich belegt v. Recklinghausen in seiner Monographie mit dem Namen "Pseudoglomeruli" eigenthümliche aus lymphadenoidem Gewebe bestehende kugelige oder halbkugelige Gebilde, welche in das Lumen der Drüsen seiner Tumoren hineinragen. Dieselben gleichen den Malpighi'schen Körperchen, „jenen eigentlichen Repräsentanten des secretorischen Abschnittes des Wolff'schen Körpers", welche zur Zeit der vorgerückten Verkümmerung dieses Organs von $\mathrm{Czerny}$ in dem Analogon des weiblichen Paroophoron, dem sogenannten Giraldès'schen Organ des Mannes, wenigstens für Säugethiere nachgewiesen wurden. Der von Cullen abgebildete Pseudoglomerulus ist indess seiner Form und namentlich seiner Grösse wegen nicht ohne weiteres mit den v. Recklinghausen'schen zu identificiren. Bei 16 facher Vergrösserung erseheint jener umfangreicher als diese bei zweihundertfacher! Das Drüsenepithel ist bei Cullen an verschiedenen Stellen auf einer Seite cylindrisch, auf der anderen fast flach. Die Pseudoglomeruli sind mit cylindrischem Flimmerepithel bedeckt. Was den Ursprung der drüsigen Elemente betrifft, so giebt Czerny wegen der grossen Uebereinstimmung mit den Befunden v. Recklinghausen's in den Adenomyomata des Uterus zu, dass sie, wie v. Recklinghausen für seine Fälle nachgewiesen hat, vom Wolff'schen Körper abstammen mögen Andererseits kann er jedoch nicht umhin, nangesichts der auffallenden Aehnlichkeit mit denen der uterinen Mucosa und der

1) Die Adenomyome und Cystadenome der Uterus- und Tubenwandung, ihre Abliunft von Resten des Wolff'schen Körpers. Berlin 1896. 
Thatsache, dass ihr Stroma dem der Mucosa ähnlich ist, die Möglichkeit anzunehmen, dass sie von einem abnormen embryonalen Depositum eines Theiles des Müller'schen Ganges herstammen".

Bei Pfannenstiel ${ }^{1}$ ) handelt es sich um einen kaum wallnussgrossen Tumor, der in der Leistengegend etwas nach innen und unten vom äusseren Leistenring gelegen und mit den aus dem Leistencanal ausstrahlenden Gewebspartien ganz diffus verschmolzen war. Ein deutliches Stück des Ligamentum rotundum kam bei der Exstirpation nicht zu Gesicht. Der Tumor zeigte die typische Structur des Adenomyoms und die kleinen Pigmentkörperchen nach v. Recklinghausen. Ein gleicher Tumor fand sich im hinteren Scheidengewölbe dem Uterus nahe anliegend. Pfannenstiel nimmt ohne weiteres an, dass der Inguinaltumor keine Metastase des Scheidentumors ist, sondern, „dass einerseits das Paroophoron etwas abnorm tief hinabragte bis zur Scheide und andererseits bei der Entwickelung der Genitalien der unterste Abschnitt der Urniere mit dem Ligamentum rotundum in die Leistengegend gelangte".

Neben diesen beiden Fällen, deren Uebereinstimmung mit dem unsrigen unverkennbar ist, mag ein dritter, der von A. Martin 1. c. als Fall II beschriebene, nicht unerwähnt bleiben, wenngleich eine Deutung als Tumor des Ljg. rot. wohl einen Zweifel zulässt. Es war eine riesige intraabdominale Geschwulst, die dem Beckeneingang auflag und nach oben bis zum Thoraxrand reichte. Sie inserirte an dem linken Lig. rot. mit einem "lockeren Gewebe, das sich vom Lig. rot. abtrennen und zu einem Stiel vereinigen liess". Der Tumor bestand aus einem grossen dünnwandigen Sack, der nach unten hin eine strangartige Verdickung zeigte. Während in der grossen Cyste an der Wand nur Detritus, Epithelien, etwas Fett und Cholestearin gefunden wurde, konnte man in dem Strang, der beim Aufschneiden aus mehreren kleinen, in einander übergehenden Cysten bestand, erkennen, dass dieselben einen hellgelben, dünnflüssigen Inhalt hatten. Nur an der Innenwand der einen fand sich ein niedriges, cylindrisches Flimmerepithel. M. meint, dass der Bau unzweifelhaft mit den von Olshausen beschriebenen Parovarialtumoren übereinstimmt. "Wie aber," fragt er, "soll man die Verlagerung des Tumors aus

1) Bericht über die Verhandlungen der VII. Versammlung der deutschen Gesellschaft für Gynäkologie etc. Centralbl. f. Gyn. 1897. No. 25. Fall II. 
dem Lig. lat. erklären, aus der Nachbarschaft des Ovarium nach dem Lig. rot. hin, ohne dass dieses an dieser Wanderung theilgenommen oder auch nur eine Spur der Verbindung übrig geblieben ist? Nachdem v. Recklinghausen auf die Kystombildung des Paroophoron aufmerksam gemacht, lässt sich die Vermuthung nicht von der Hand weisen, es möge sich hier um ein Paroophoralkystom, das autochthon, d. h. an physiologischer Stelle entstanden und erst secundär - es bestanden Verwachsungen mit der Bauchwand und den Därmen - mit dem Lig. rot. in Verbindung getreten sei, gehandelt haben. Ist doch in neuester Zeit von L. Pick ${ }^{1}$ ) "Ein neuer Typus des voluminösen paroophoralen Adenomyoms" geschildert worden, welcher das Vorkommen betrüchtlich grosser Cysten des Paroophoron beweist. Es wäre Martin's Tumor demnach ein juxtanterines voluminöses Adenokystom ohne Myombildung! Freilich lehnt v. Recklinghausen eine autochthone intraligamentäre Entstehung von Adenocysten ab, da er "geradezu ein Auswanderungsbestreben kleinerer subseröser Adenomyome und Cysten in das parametrale Bindegewebe und in Adhäsionen nachweisen konnte $\left.{ }^{2}\right)^{\text {", }}$, und auch Pick nimmt für das parametrale Adenomyom von Breus $^{3}$ ), das er entgegen dem Autor nicht vom Gartner'schen Gang, sondern vom Wolff'schen Körper ableitet, eine secundäre parametrale Verpflanzung aus dem Myometrium an. In seinem eigenen Fall stand der Tumor mit der Uterusfläche in "lockerer, leicht löslicher Verbindung". Es liesse in Analogie hierzu Martin's Fall auch die Deutung zu, dass es sich in der That um einen Tumor des Lig. rot. gehandelt, d. h. dass das ligament paroophorale Keime eine Strecke weit mitgeschleppt habe und diese bei ihrem Auswachsen zum Kystom aus dem Ligament derart herausgewachsen seien, dass nur noch eine lockere Verbindung mit dem letzteren übrig geblieben.

Eine weitere Vermuthung ist die des abgesprengten Ovarialkeimes mit folgender Kystomentwickelung.

Kehren wir zu unserem Fall zurück und prüfen wir ihn auf seine Aehnlichkeit mit den Befunden v. Recklinghausen's, so

1) Arch. f. Gynäk. Bd. 54. H. 1.

2) s. Pick, l. c.

3) Breas, Ueber wahre Epithel führende Cystenbildung in Uterusmyomen (Fall I). Wien 1894. 
lassen unsere Bilder ebenso wie bei $\mathrm{Cullen}^{1}$ ) jene systematische Gruppirung der Drüsen, wie v. Recklinghausen sie beschreibt, vermissen. Wir haben keine scharfe Scheidung in Secretions- und Sammelröhrchen, keine gänsemarschartige Anordnung, keine Schleifen und auch keine Pseudoglomeruli. Dagegen lassen einmal die an gewissen Stellen deutlich hervortretenden Anhäufungen "cystogenen" Gewebes und vielleicht die halbmondförmigen Drüsen einen Vergleich mit v. Recklinghausen's Befunden zu. Diese Drüsen würden alsdann .v. Recklinghausen's Ampullen entsprechen. Wie diese zeigen sie auf ihrem Dache (convexe Seite) ein niedrigeres Epithel als auf dem Boden; wie bei diesen ist der Boden hergestellt durch ein rundzellen- und gefässreiches, cytogenes Gewebe. Beide umsehliessen nicht selten grosse, fast kugelige, gelblichglänzende körnchen- und kernhaltige Zellen, weisse und rothe Blutkörperchen, letztere abgeblasst, selbst ganz entfärbt. Endlich findet sich bei beiden „Blutaustritt in die obersten Schichten, namentlich in den flachen Erhebungen auf dem Boden". Vielleicht könnte man diese Bodenerhebungen, die wir ausser in den halbmondförmigen Drüsen auch einmal zu zweien in einer schlauchförmigen fanden, in Beziehung bringen zu v. Recklinghausen's Pseudoglomeruli. Denn wenn er den letzteren auch gern eine secretorische Rolle zuschreiben möchte, so kann er doch, da er in den Adenomen keine morphologischen Elemente, welche eine Secretion bewiesen hätten, aufgefunden hat, „den Gedanken, dass die genannten Pseudoglomeruli nur als Lymphknötchen zu betrachten sind, nicht von der Hand weisen". In unserm Fall scheinen die in der Umgebung der Drüsen befindlichen Anhäufungen von lymphadenoidem Gewebe im Verein mit den ähnlichen Bildungen an den Lymphspalten kaum eine andere Deutung zuzulassen, während die die Gefässe begleitenden Rundzellen wohl entzündlicher Natur sind. Wenn wir ausser den halbmondförmigen auch unsere im Querschnitt kreisrund erscheinenden Drüsen in den Bildern v. Recklinghausen's wiederfinden, so lässt sich doch andererseits nicht leugnen, dass dieselben eine so grosse Aehnlichkeit mit den Uterusdrüsen zeigen, dass sie leicht mit diesen verwechselt werden können. Ebensowenig wie Cullen können deshalb auch wir allein nach dem mikroskopischen Befunde ein entscheidendes Urtheil über

1) Anmerk.: Bei Pfannenstiel's Fall II ist hierüber nichts Ausdrückliches vermerkt. 
den Ursprung der drüsigen Elemente, ob sie vom Wolff'schen Körper oder vom Müller'schen Gange stammen, fällen. Giebt doch auch $\nabla$. Recklinghausen für seine anscheinend so wohlcharakterisirten Bilder zu, "dass in jedem Beobachter, bei der flüchtigen Betrachtung unmittelkar die Vorstellung erweckt wird, es läge Uterusschleimhaut vor", und konnte doch $\mathrm{Pick}^{1}$ ) das "allgemeine Gesetz" aufstellen, "dass zwischen neoplastischen Abkömmlingen des Mesonephros und der Müller'schen Gänge sich augenfällige Structuranalogien herausbilden können". "Hatte früher, fährt er fort, die Morphologie der drüsigen Elemente der Adenomyome die genetischen Vorstellungen in der Adenomyomfrage stets von neuem anscheinend beweiskräftig auf die Müller'schen Fäden gelenkt, so wissen wir eben jetzt, dass jene Congruenz ebenso wenig zu Gunsten der Abstammung der Adenomschläuche vom Endometrium des Gebärmutterkörpers wie gegen die vom Wolffschen Körper in's Feld geführt werden kann".

Wir müssen uns deshalb nach einem anderen Wege umsehen, auf welchem wir im vorliegenden Fall zur Klarheit in dieser Frage gelangen können.

Von Wieger ${ }^{2}$ ) erfahren wir, dass das Ligamentum rotundum des Weibes thatsächlich ein Analogon des Gubernaculum Hunteri des Mannes ist. Seine Untersuchungen zehnwöchentlicher Embryonen ergaben, dass das Ligament ausgeht vom Parovarium (Rest des Wolff'schen Körpers) und sich von hier zum Ovarium begiebt, an dessen innerer und hinterer Seite es in einer Rinne als ein Zug von glatten Muskelfasern verläuft. Aus dieser Rinne tritt es als kurzer, straffer Strang (Lig. ovarii) aus, um sich an die Tube anzusetzen. „Bei Loupenvergrösserung zeigt es sich aber, dass dieses Band an seiner Ansatzstelle an der Tube nicht aufhört, sondern dass man auf der Tube den Wulst verfolgen kann, welchen dieser Strang auf ihrer dorsalen Seite bildet. Man sieht deutlich, dass sich der Strang des Ligamentum ovarii mitten auf der Tube unter einem Winkel von ca. $120^{\circ}$ gegen seine bisherige Richtung abknickt, und dass er sich dann erst an der dorsalen Seite der Tube noch festsitzend und gleich darauf frei geworden

1) 1. c. S. 120 .

2) Ueber die Entstehung und Entwicklung der Bänder des weiblichen Genitalapparates beim Menschen. Archiv f. Anatomie u. Physiologie. Anat. Abthl. 1885. 
656 Bluhm, Zur Pathologie des Ligamentum rotundum uteri.

(mit etwas stärkerem Caliber) weiter fortsetzt als Ligamentum teres".

Das Ligamentum rotundum nimmt also seinen Ausgangspunkt vom Wolff'schen Körper und tritt erst secundär mit dem Müllerschen Gange in Verbindung. Ist es da nicht sehr wahrscheinlich, dass es jene Drüsenkeime von seinem Ursprungsort mitschleppt und sie nicht erst unterwegs bei seiner Begegnung mit anderen Organen von diesen sozusagen aufgelesen hat?

v. Recklinghausen hebt die grosse Häufigkeit der Tubenwinkeladenomyome hervor. Er allein verfügt über 30 Beobachtungen gegenüber 11 von Uterusadenomyomen. Dabei fanden sich die Tumoren meist excentrisch auf der Dorsalwand der Tube. Am Tubenwinkel inserirt das Ligamentum rotundum und zwar legt es sich der Tube, wie wir von Wieger gehört haben, auf deren dorsalen Seite an. Muss man unter diesen Verhältnissen nicht daran denken, dass dieses Ligament eine Rolle beim Transport der Adenomyomkeime gespielt hat?

Ich glaube daher nicht fehlzugehen, wenn ich nicht sowohl aus der Aehnlichkeit des mikroskopisohen Befundes, als vielmehr aus der Entwickelung des Ligamentum rotundum den Schluss ziehe, dass es sich in meinem Fall, dem Fall von Cullen und demjenigen von Pfannenstiel thatsächlich um Abkömmlinge des Wolff'schen Körpers handelt entsprechend der Auffassung $v$. Recklinghausen's für die dorsalständigen Adenomyome des Uterus und der Tubenwinkel.

Wir haben es nach alledem in unserem Fall zu thun mit einem lymphangiektatischen Cystofibrom des Jigamentum rotundum uteri mit Einschlüssen drüsiger Abkömmlinge des Wolff'schen Körpers. 
Bluhm, Zur Pathologie des Ligamentum rotundum uteri.

\section{Erklärung der Abbildungen auf Tafel XXVII.}

Figur 1. Uebersichtsschnitt bei 20 facher Vergrösserung.

L u. L' Lymphräume.

D Drüsen.

B Zellarme bindegewebige Grund̀substanz des Tumors.

$\mathrm{R}$ Rundzellenhanfen an den Lymphräumen (Capilaren, freie Blutkörperchen und zum Theil sehr reichliches Pigment umschliessend.)

cy Anhäufungen von cytogenem Gewebe an den Drüsen.

$P$ Pigment.

Figur 2. Halbmondförmige Drüse bei 85 facher Vergrösserung.

D Drüse.

cy Cytogenes Gewebe.

B Fibrilläres Bindegewebe.

Figur 3. Gruppe unregelmässig geformter Drüsen, 60 fach vergrössert.

D Drüsen.

B Fibrilläres Bindegewebe.

cy Cytogenes Gewebe mit rothen Blutkörperchen und Pigmentzellen. 